In der Rubrik „Literatur kompakt" werden die wichtigsten Originalarbeiten aus der internationalen Fachliteratur referiert.

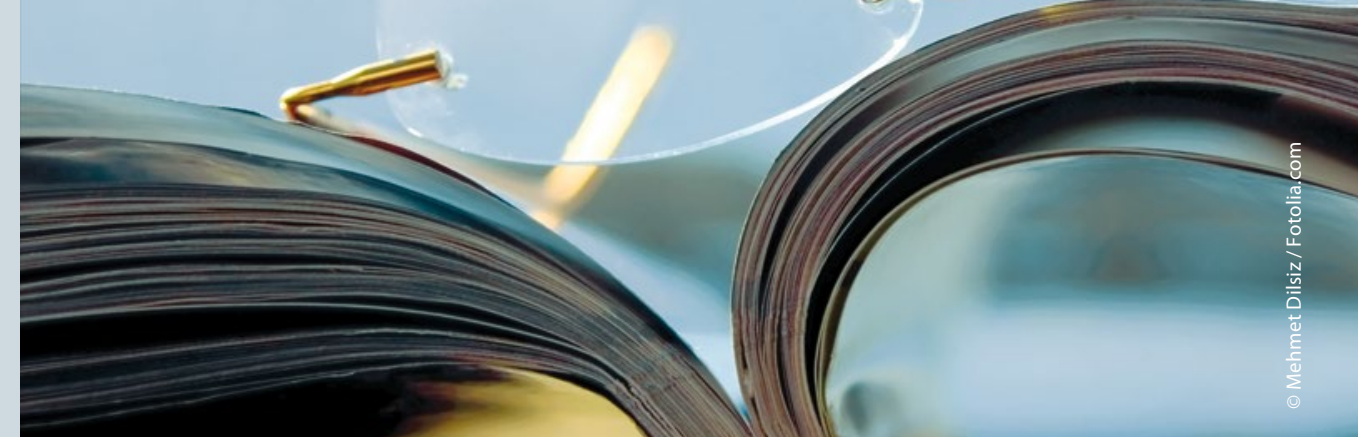

\section{Prostatakarzinom: Überlebensvorteil durch viel Vitamin D im Blut?}

\begin{abstract}
Hohe Calcidiol-Spiegel im Blut vor der Diagnose Prostatakrebs sind offenbar mit einem längeren Überleben der krebskranken Männer assoziiert. Das ist unabhängig vom Alter der Patienten und dem Tumorstadium zum Zeitpunkt der Diagnose sowie von der körperlichen Fitness.
\end{abstract}

\begin{abstract}
alcidiol (25-OH-Vitamin D) spiegelt am besten die Vitamin-D3-Versorgung im Blut innerhalb der vergangenen drei bis vier Monate wider. Da es bisher widersprüchliche Ergebnisse zum Zusammenhang zwischen Vitamin D3 im Blut und der Entwicklung eines Prostatakarzinoms gab beziehungsweise entsprechende Studien wenig aussagekräftig waren, haben die Krebsepidemiologin Dr. Alison M. Mondul von der University of Michigan School of Public Health in Ann Arbor und ihre Kollegen Daten der US-amerikanisch-finnischen ATBC-Studie (Alpha-Tocopherol, BetaCarotene Cancer Prevention) in Hinblick auf diesen Aspekt ausgewertet.

In der ATBC-Studie, die 1985 begonnen hatte, wurde der primärpräventive
\end{abstract}

Effekt von Alpha-Tocopherol und Betacarotin bei mehr als 23.000 gesunden Rauchern in Finnland überprüft. Supplementiert wurde über einen Zeitraum von fünf bis acht Jahren. Die Dokumentation der Follow-up-Daten endete im Dezember 2012. Für die Studie von Mondul et al., in der auf die Laborwerte jeweils einer prospektiv entnommenen Nüchternblutprobe zurückgegriffen wurde, werteten die Epidemiologin und ihre Kollegen die Befunde von 1.000 Teilnehmern aus, die bis zu diesem Zeitpunkt an einem Prostatakarzinom erkrankt waren. Die Todesursachen wurden anhand des finnischen Sterberegisters ermittelt. Bis Ende 2012 starben 363 der Studienteilnehmer an den Folgen des Prostatakarzinoms.

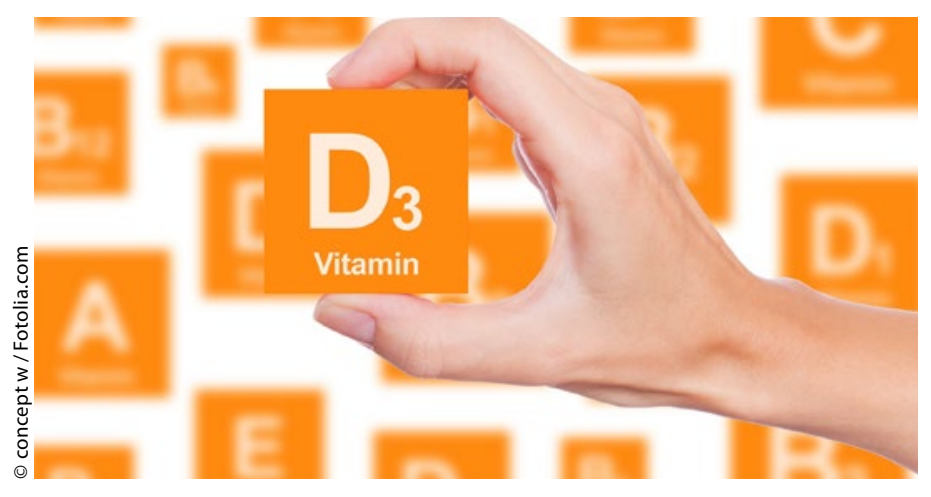

Wer die Überlebenszeit bei Prostatakarzinomen einschätzen will, sollte den Calcidiolanteil im Blut der Patienten beachten.
Wie die Epidemiologen berichten, war die Wahrscheinlichkeit, an Prostatakrebs zu sterben, für Patienten mit den höchsten 25-OH-Vitamin-D-Spiegeln signifikant niedriger als für Männer mit den niedrigsten Werten, und zwar um $31 \%$ (Hazard Ratio [HR]: 0,69; 95\%-Konfidenzintervall zwischen 0,50 und 0,95; p-Trend: 0,003). Bei der Berechnung hatten die Wissenschaftler das Alter der Männer zum Zeitpunkt der Krebsdiagnose berücksichtigt. Im Winter lagen die Vitamin-D-Werte bei über 45 versus $\leq 16 \mathrm{nmol} / \mathrm{l}$, im Sommer bei über 60 versus $\leq 26 \mathrm{nmol} / \mathrm{l}(1 \mathrm{nmol} / \mathrm{l}$ $=0,401 \mu \mathrm{g} / \mathrm{l})$. Nur wenig verändert war das relative Sterberisiko, wenn Mondul und ihre Kollegen eine Multivariatanalyse vornahmen, in der auch die körperliche Fitness und die Familienanamnese berücksichtigt wurden (HR: 0,72; 95\%-Konfidenzintervall zwischen 0,52 und 0,99; p-Trend: 0,006).

Fazit: Bei der Analyse fiel den Epidemiologen auf, dass der beobachtete positive Zusammenhang, der unabhängig vom Stadium der Erkrankung und vom Tumorgrad bei der Diagnose war, sich nur bei jenen Patienten als signifikant herausstellte, die mehr als drei Jahre überlebt hatten (HR: 0,95 bei Überleben $<3,3$ Jahre seit der Diagnose versus HR: 0,53 bei längerem Überleben). Aufgrund dieser Tatsache sei die Wahrscheinlichkeit für eine Verzerrung durch ein LeadTime-Bias, also ein scheinbar längeres Überleben bei einer frühen Krebsdiagnose, eher gering.

Peter Leiner

Mondul AM et al. Circulating 25-hydroxyvitamin D and prostate cancer survival. CEBP. 2016; doi:10.1158/1055-9965.EPI-15-0991. 